\title{
Duração da luminosidade e tamanho de recipientes para a produção de alface em hortas urbanas
}

\author{
Antonio Alves Pinto ${ }^{1}$, Laudeline Dantas Santana ${ }^{1}$, Felipe Thomaz da Camar ${ }^{1}$, Wíctor Állyson \\ Dias Rodrigues ${ }^{1}$, Francisco Edson da Silva ${ }^{1}$
}

${ }^{1}$ Universidade Federal do Cariri, Juazeiro do Norte, Ceará, Brasil. E-mail: antonioalvesaap01@ gmail.com, laudelinedantas@gmail.com, felipe.camara@ufca.edu.br, wictor.allyson@hotmail.com, pintoalves01@hotmail.com

Recebido: 17/04/2017; Aceito: 12/06/2018.

\section{RESUMO}

A necessidade de consumo de alimentos de melhor qualidade tem conduzido à produção de hortaliças em ambientes domésticos, prática que tem sido denominada de hortas urbanas. Neste estudo foram avaliados os efeitos do volume do recipiente e da duração da luminosidade direta no crescimento e no rendimento das plantas de alface. O trabalho foi realizado no Centro de Ciências Agrárias e da Biodiversidade (CCAB) da Universidade Federal do Cariri (UFCA). O delineamento experimental utilizado foi inteiramente casualizado, em esquema fatorial $2 \times 4$, com três repetições. Os tratamentos constituíram de dois tempos da duração da luminosidade (4 e 7 horas de sol) e de três tamanhos de recipientes $\left(0,6 ; 1,2 ; 1,8\right.$ e $\left.3,4 \mathrm{dm}^{3}\right)$. Foram conduzidos dois experimentos e duas épocas distintas, sendo em ambos os experimentos avaliados a fitomassa das plantas, das folhas, do caule e das raízes, o diâmetro da parte aérea e caule e o comprimento do caule e das raízes. O melhor crescimento e produtividade de alface foram verificados com a utilização de recipientes com maior volume $\left(3,4 \mathrm{dm}^{3}\right)$. A duração da luminosidade teve pouco efeito no crescimento das plantas de alface, indicando que esta cultura pode ser cultivada em locais com apenas $4 \mathrm{~h}$ de luminosidade direta sobre as plantas.

Palavras-chave: volume do recipiente, luz direta, Lactuca sativa

\section{Luminosity duration and container size for the production of lettuce in urban vegetable gardens}

\begin{abstract}
Currently, the need for better food consumption has led to the production of vegetables in domestic environments, with this practice being called urban vegetable gardens. Therefore, the objective of this work was to evaluate the development and productivity of lettuce as a function of container size and luminosity on lettuce plants, for the implantation of urban vegetable gardens. The work was carried out at the Centro de Ciências Agrárias e da Biodiversidade (CCAB) of the Universidade Federal do Cariri (UFCA). The experimental design was completely randomized, in a $2 \times 4$ factorial scheme, consisting of three replicates. The first factor analyzed was sunlight duration (4 and 7 sunlight hours) and the second were the four container sizes $\left(0,6 ; 1,2 ; 1,8\right.$ e $\left.3,4 \mathrm{dm}^{3}\right)$. The experiment was conducted in two cycles, and the plant biomass, leaf biomass, stem biomass and root biomass were evaluated; besides the aerial part diameter, stem diameter, and stem and root length. There was a better development and productivity for the use of containers with higher volume $\left(3.4 \mathrm{dm}^{3}\right)$ and little effect of the sunlight duration, demonstrating that lettuce can be grown in residences with only $4 \mathrm{~h}$ of direct luminosity on the crop.
\end{abstract}

Keywords: container volume, insolation, Lactuta sativa 


\section{Introdução}

A procura por hortaliças de qualidade, sem uso de defensivos agrícolas, tem aumentado consideravelmente nos últimos anos, o que tem induzido muitas pessoas a produzirem sua própria hortaliça nas residências. $\mathrm{Na}$ zona rural é comum a população produzir as hortaliças que consomem, pois, o custo é baixo, e o espaço é amplo. Na zona urbana, a implantação de hortas domésticas vem crescendo com o cultivo de hortaliças que não necessitam de grandes áreas para se desenvolver (Monteiro e Mendonça, 2004).

Diferentemente da zona rural, a produção de hortaliças nas áreas urbanas é realizada utilizando-se vasos ou recipientes recicláveis. A alface (Lactuca sativa L.) possui sistema radicular curto, chegando à 25 $\mathrm{cm}$ de profundidade, desde que seja transplantada, sendo favorecida por temperaturas amenas (Filgueira, 2013); constitui como opção para ser cultivada em vasos e em locais de pouca radiação luminosa, como nas residências de centros urbanos.

A alface é uma hortaliça folhosa que não tolera temperaturas altas, por tal motivo tem seu cultivo limitado na região Nordeste (Moreira et al., 2014). No entanto, as telas de sombreamento ou casas de vegetação tem sido empregadas com êxito no cultivo desta hortaliça, reduzindo a incidência de luz na planta e proporcionando um ambiente adequado para melhor qualidade das folhas e maior produtividade (Diamante et al., 2013).

As condições climáticas de algumas regiões, como temperaturas muito elevadas ou muito baixas, podem conduzir a modificações morfofisiológicas, influenciando diretamente na produtividade da cultura (Blind e Silva-Filho, 2015). O cultivo em áreas onde a luz solar não incide o dia todo, é uma alternativa para plantar alface sem necessidade de telados e tem apresentado bons resultados. Porém, nas residências, a falta de luz pode ser um problema para o desenvolvimento da alface devido aos muros altos e pouco espaço.

No entanto, as plantas possuem algumas habilidades para se adaptarem ao ambiente onde se encontram, seja este com maior ou menor luminosidade (Holt, 1995). A resposta ao seu desenvolvimento está relacionada à habilidade de adaptação ao ambiente (Dias-Filho, 1997).

Diante do exposto, o objetivo deste trabalho foi avaliar o crescimento e a produtividade de alface em função do volume do recipiente e da duração da luminosidade para implantação de hortas urbanas.

\section{Material e Métodos}

O trabalho foi realizado no Centro de Ciências Agrárias e da Biodiversidade (CCAB) da Universidade Federal do Cariri (UFCA), localizado no município de
Crato, Ceará, com as coordenadas geográficas $7^{\circ} 14^{\prime} 49^{\prime \prime} \mathrm{S}, 39^{\circ} 22^{\prime} 05^{\prime \prime} \mathrm{W}$ e $413 \mathrm{~m}$ de altitude, onde apresenta um clima entre Tropical Semiárido à Tropical Semiárido Brando, com temperatura média entre 24 a $26{ }^{\circ} \mathrm{C}$ e período chuvoso de janeiro a maio. A média pluviométrica é de 925 mm (Lima e Ribeiro, 2012).

Os dados de temperatura no período de 26 de dezembro de 2016 à 10 de março de 2017 estão na Figura 1, sendo referentes ao período de condução dos dois ciclos da cultura de alface.

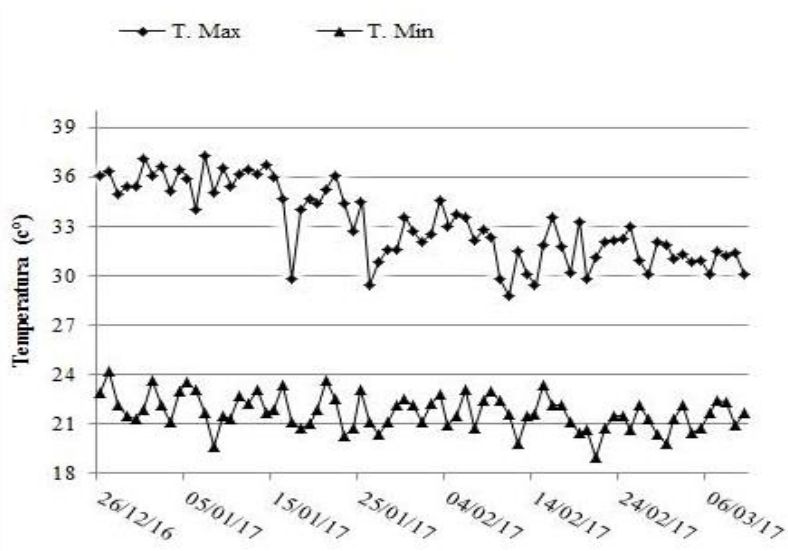

Figura 1. Temperatura máxima e mínima do ar diária $\left({ }^{\circ} \mathrm{C}\right)$, no período de 26/12/2016 à 10/03/2017, dados adaptados do Inmet (2017).

O delineamento experimental utilizado foi inteiramente casualizado, em esquema fatorial $2 \times 4$, com três repetições. O primeiro fator analisado foi constituído pela duração da luminosidade incidente nas plantas (4 e 7 horas de sol) e o segundo fator foi constituído por quatro volumes dos recipientes $\left[0,6 \mathrm{dm}^{3}\right.$ (10 cm de diâmetro e $8 \mathrm{~cm}$ de altura); $1,2 \mathrm{dm}^{3}(10 \mathrm{~cm}$ de diâmetro e $15 \mathrm{~cm}$ de altura); $1,8 \mathrm{dm}^{3}(10 \mathrm{~cm}$ de diâmetro e $23 \mathrm{~cm}$ de altura); e $3,4 \mathrm{dm}^{3}$ (16 cm de diâmetro e $17 \mathrm{~cm}$ de altura)].

Foram conduzidos dois experimentos em duas épocas distintas, em ambos os experimentos as mudas foram produzidas em bandejas com 128 cédulas e transplantadas 18 dias após a semeadura (DAS) nos recipientes estudados. No primeiro experimento as plantas permaneceram nos recipientes entre o dia 26 de dezembro de 2016 e 26 de janeiro de 2017; no segundo experimento entre 8 de fevereiro e 10 de março de 2017. Portanto, o primeiro experimento teve duração de 31 dias nos recipientes (total de 49 DAS) e o segundo 30 dias nos recipientes (48 DAS).

Todos os recipientes avaliados foram preenchidos com substrato contendo $50 \%$ de solo e $50 \%$ de composto orgânico. O solo foi classificado como Argissolo Vermelho-Amarelo, conforme classificação Funceme (2012). A composição química foi: $\mathrm{pH}$ (1:2,5 $\mathrm{H}_{2} \mathrm{O}$ ): 6,0; $\mathrm{P}$ (Mehlich-1): 3,0 $\mathrm{mg} \mathrm{dm}^{-3} ; \mathrm{K}: 1,27 \mathrm{mmol}_{\mathrm{c}}$ $\mathrm{dm}^{-3}$; Ca: 5,0 $\mathrm{mmol}_{\mathrm{c}} \mathrm{dm}^{-3}$; $\mathrm{Mg}: 6,0 \mathrm{mmol}_{\mathrm{c}} \mathrm{dm}^{-3}$; CTC: 
$35,4 \mathrm{mmol}_{\mathrm{c}} \mathrm{dm}^{-3}$ e saturação por bases de $53 \%$. O composto foi produzido a partir da proporção de três partes de restos culturais de sorgo sacarino para uma parte de esterco bovino. A constituição química do composto, com base em massa seca, foi: $\mathrm{N}$ : $0,8 \%$; $\mathrm{P}$ : 0,1\%; K: 0,2\%; Ca: 0,02\%; Mg: 0,11\% e MO: $15,6 \%$.

No segundo experimento foram utilizados os mesmos substratos do experimento 1 , sem ocorrer a substituição por um novo substrato, fato comum em hortas urbanas, visando reaproveitar o mesmo substrato. Foram efetuadas regas diárias desde a semeadura até a colheita, no período da manhã.

A colheita da alface, para análise, foi realizada quando as plantas começaram a tender ao pendoamento. As variáveis analisadas foram o diâmetro da parte aérea, o comprimento e a largura da maior folha, o comprimento do caule e da raiz, com auxílio de uma fita métrica. Para o diâmetro do caule, utilizou-se um paquímetro. O número de folhas foi determinado pela contagem de todas as folhas, descartando as menores, localizadas na extremidade do caule. As massas da planta inteira, das folhas, do caule e da raiz, todas foram mensuradas em balança semianalítica de precisão para duas casas decimais, em gramas.

Os dados foram submetidos à análise de variância, com o volume do recipiente sendo submetido à análise de regressão para determinação do melhor modelo significativo. Utilizou-se o programa estatístico SISVAR 5.3 (Ferreira, 2011).

\section{Resultados e Discussão}

Os resultados da análise de variância reportaram que não houve interação significativa entre os fatores, no primeiro ciclo, para a massa da planta e das folhas, todavia, para o diâmetro da parte aérea e todas variáveis no segundo ciclo ocorreram interações significativas (Tabela 1). Verifica-se na Tabela 1, que a massa das plantas e das folhas não obtiveram diferenças significativas $(p>0,05)$ para a luminosidade direta, ambas no primeiro ciclo. Resultados semelhantes aos de Silva et al. (2015), ao avaliarem o desempenho agronômico de alface, sob diferentes níveis de sombreamento, obtiveram média de massa de planta de 95,6 g para o sombreamento com espaldeira e de 87,2 $\mathrm{g}$ sem sombreamento, porém, não significativo.

Dalastra et al. (2016) ao estudarem o desempenho produtivo de alface, cultivada sob telado em níveis diferentes de sombreamento, observaram maiores médias de massa da planta sem sombreamento (171,3 g) em relação ao uso de telado com $50 \%$ de sombreamento (119 g), com tais resultados, no campo, sendo superiores aos encontrados, nos recipientes, neste trabalho.

No segundo ciclo ocorreu interação significativa para as variáveis MP e MF, enquanto que o diâmetro da parte aérea obteve interação significativa para os dois ciclos (Tabela 1). Os desdobramentos destas interações, para avaliar o efeito da luminosidade em cada volume de recipiente estudado, estão nas Tabelas 2 e 3, respectivamente; a massa da planta foi semelhante estatisticamente.

Observa-se na Tabela 3 e Figura 2, o desdobramento da interação entre os fatores estudados. No $1^{\circ}$ ciclo, as plantas do recipiente com $0,6 \mathrm{dm}^{3}$ obtiveram maior diâmetro aéreo quando expostas a uma luminosidade de $4 \mathrm{~h}$, enquanto que no segundo ciclo as plantas cultivadas nos recipientes com $3,4 \mathrm{dm}^{3}$ e com luminosidade de $4 \mathrm{~h}$, obtiveram maior diâmetro da parte aérea. Para os recipientes com 1,2 e $1,8 \mathrm{dm}^{3}$ os resultados foram semelhantes entre os períodos de luminosidade avaliados.

Tabela 1. Resultado da análise de variância e valores médios do fator luminosidade para a massa das plantas (MP), a massa das folhas (MF) e o diâmetro da parte aérea (DA) para o primeiro e segundo ciclo da alface.

\begin{tabular}{|c|c|c|c|c|c|c|}
\hline \multirow{3}{*}{ Fontes de variação } & \multicolumn{6}{|c|}{ Valores de F } \\
\hline & \multicolumn{2}{|c|}{ MP } & \multicolumn{2}{|c|}{ MF } & \multicolumn{2}{|c|}{ DA } \\
\hline & $1^{\circ} \mathrm{Ciclo}$ & $2^{\circ}$ Ciclo & $1^{\circ}$ Ciclo & $2^{\circ}$ Ciclo & $1^{\circ}$ Ciclo & $2^{\circ}$ Ciclo \\
\hline Luminosidade (L) & $1,06^{\mathrm{NS}}$ & $2,54^{\mathrm{NS}}$ & $0,61^{\mathrm{NS}}$ & $4,13^{\mathrm{NS}}$ & $7,62 * *$ & $4,85 *$ \\
\hline Recipiente $(\mathrm{R})$ & $8,23 * *$ & $21,31 * *$ & $15,09 * *$ & $21,53 * *$ & $35,44 * *$ & $15,03 * *$ \\
\hline Interação (L*R) & $0,54^{\mathrm{NS}}$ & $16,80 * *$ & $0,95^{\mathrm{NS}}$ & $12,17 * *$ & $4,37 *$ & $10,67 * *$ \\
\hline \multirow[t]{2}{*}{$\mathrm{CV} \%$} & 54,52 & 20,58 & 48,37 & 21,37 & 11,51 & 9,67 \\
\hline & \multicolumn{6}{|c|}{ Valor Médio } \\
\hline \multirow{3}{*}{ Luminosidade (h) } & \multicolumn{2}{|c|}{ MP } & \multicolumn{2}{|c|}{ MF } & \multicolumn{2}{|c|}{ DA } \\
\hline & $1^{\circ} \mathrm{Ciclo}$ & $2^{\circ}$ Ciclo & $1^{\circ} \mathrm{Ciclo}$ & $2^{\circ}$ Ciclo & $1^{\circ}$ Ciclo & $2^{\circ}$ Ciclo \\
\hline & \multicolumn{2}{|c|}{------ g ------- } & \multicolumn{2}{|c|}{--------- g ----------- } & \multicolumn{2}{|c|}{-------- cm --------- } \\
\hline 4 & 37,7 & - & 30,0 & - & - & - \\
\hline 7 & 47,5 & - & 35,1 & - & - & - \\
\hline
\end{tabular}


Tabela 2. Interação entre os fatores volume do recipiente e luminosidade para a variável massa das plantas e das folhas (g) no segundo ciclo da alface.

\begin{tabular}{cccc}
\hline \multirow{2}{*}{ Variável } & Recipiente & \multicolumn{2}{c}{ Luminosidade } \\
\cline { 3 - 4 } & $\left(\mathrm{dm}^{3}\right)$ & $4 \mathrm{~h}$ & $7 \mathrm{~h}$ \\
\hline \multirow{3}{*}{ Massa das } & 0,6 & $23,0 \mathrm{a}$ & $36,3 \mathrm{a}$ \\
plantas & 1,2 & $56,4 \mathrm{~b}$ & $113,3 \mathrm{a}$ \\
& 1,8 & $62,7 \mathrm{a}$ & $85,4 \mathrm{a}$ \\
& 3,4 & $114,8 \mathrm{a}$ & $58,7 \mathrm{~b}$ \\
\cline { 2 - 4 } & Média & $64,2 \mathrm{~A}$ & $73,4 \mathrm{~A}$ \\
\hline \multirow{3}{*}{ Massa das } & 0,6 & $18,1 \mathrm{a}$ & $25,5 \mathrm{a}$ \\
folhas & 1,2 & $47,2 \mathrm{~b}$ & $90,1 \mathrm{a}$ \\
& 1,8 & $46,3 \mathrm{~b}$ & $68,1 \mathrm{a}$ \\
& 3,4 & $83,0 \mathrm{a}$ & $48,9 \mathrm{~b}$ \\
\cline { 2 - 4 } & Média & $48,7 \mathrm{~A}$ & $58,1 \mathrm{~A}$
\end{tabular}

Médias seguidas pela mesma letra nas linhas, não diferem estatisticamente entre si segundo o teste de Tukey a 5\% de probabilidade.

Tabela 3. Interação entre os fatores volume do recipiente e luminosidade para a variável diâmetro da parte aérea $(\mathrm{cm})$ no primeiro e segundo ciclo da alface.

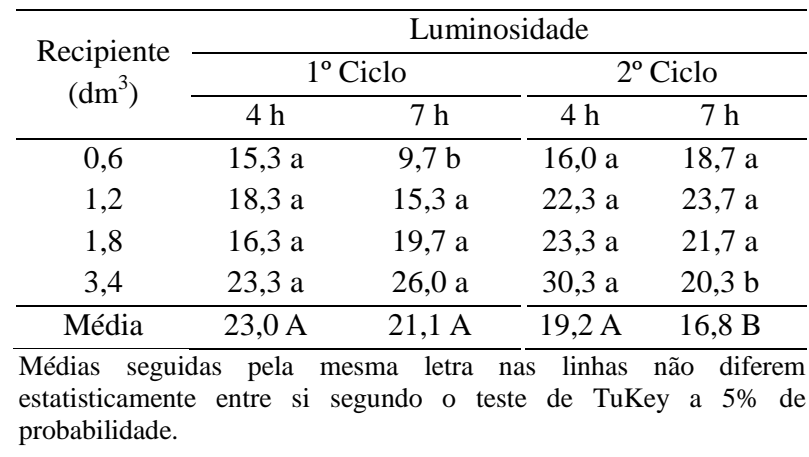

Santana et al. (2009) ao estudarem o efeito de tipos de telas de sombreamento sobre a produção e desenvolvimento de alface obtiveram médias de diâmetro aéreo de 24 e $24,3 \mathrm{~cm}$, sem e com $50 \%$ de sombreamento, respectivamente. Valores inferiores aos de Dalastra et al. (2016) que observaram média de diâmetro de 29,3 e $38,8 \mathrm{~cm}$, sem e com $50 \%$ de sombreamento, respectivamente. Todos estes resultados conduzidos em canteiros, com volume amplo de solo para as raízes explorarem, porém com resultados semelhantes aos obtidos para o maior recipiente $(3,4$ $\mathrm{dm}^{3}$ ) no primeiro ciclo, e no segundo ciclo com $4 \mathrm{~h}$ de luminosidade, demonstrando a viabilidade de produção da cultura em recipientes maiores.

Para o volume do recipiente (Figuras 2A e 2B), nota-se que a massa da planta e das folhas obteve resultados semelhantes, com a média do primeiro ciclo, e o segundo ciclo com $4 \mathrm{~h}$ de luminosidade, tendo comportamento linear, evidenciando que o aumento do recipiente proporcionou maiores valores de massa. No segundo ciclo, com $7 \mathrm{~h}$ de luminosidade, o modelo de regressão foi o polinomial de segunda ordem, apresentando diferença significativa $(p<0,01)$, com maiores valores de massa das plantas $(106,2 \mathrm{~g})$ e das folhas (85,8 g), para o recipiente com $2,1 \mathrm{dm}^{3}$.

Para o diâmetro aéreo (Figura $2 \mathrm{C}$ ), no primeiro ciclo, com 4 e $7 \mathrm{~h}$ de luminosidade, e as plantas do segundo ciclo com 4 horas de luminosidade, o melhor modelo de regressão foi o linear, significativo a $1 \%$, com o aumento do volume promovendo maior diâmetro aéreo. Enquanto que no diâmetro da parte aérea do segundo ciclo, com 7 horas de luminosidade, o modelo de regressão foi o polinomial de segunda ordem, apresentando diferença significativa $(\mathrm{p}<0,01)$, com maior diâmetro $(23,1 \mathrm{~cm})$ para o recipiente com volume de $2,1 \mathrm{dm}^{3}$. Na Tabela 4, o coeficiente de variação, conforme Pimentel Gomes (2009) foi muito alto para o $1^{\circ}$ ciclo na variável comprimento da maior folha, médio para o $2^{\circ}$ ciclo das variáveis $\mathrm{CF}, 1^{\circ}$ e $2^{\circ}$ ciclo da $\mathrm{LF}$, segundo ciclo da variável número de folhas e alto para $\mathrm{o}$ $1^{\circ}$ ciclo do número de folhas.

Na Tabela 4, observa-se que não houve diferença estatística significativa para o fator luminosidade, no primeiro ciclo, para a variável comprimento da maior folha e número de folhas, enquanto que Silva et al. (2014) verificaram influência direta da luminosidade no número de folhas. Para o $\mathrm{CF}\left(2^{\circ}\right.$ ciclo), a $\mathrm{LF}\left(1^{\circ}\right.$ e $2^{\circ}$ ciclos) e o $\mathrm{NF}\left(2^{\circ}\right.$ ciclo $)$ ocorreram interações significativas com tais interações sendo desdobradas nas tabelas 5 e 6 . Na tabela 5, para o segundo ciclo, os recipientes com $3,4 \mathrm{dm}^{3}$ proporcionaram maior comprimento da folha e número de folhas para as plantas que foram expostas à luminosidade de quatro horas, com os demais recipientes não sofrendo influência significativa do número de horas de luminosidade direta. Estas variáveis são importantes indicativos de qualidade, pois segundo Flôres et al. (2016), plantas mais enfolhadas são valorizadas pelos consumidores.

No $1^{\circ}$ ciclo, o recipiente com $3,4 \mathrm{dm}^{3}$ e luminosidade de $7 \mathrm{~h}$, proporcionou aumento na largura da folha (Tabela 6), resultado também observado no segundo ciclo para os recipientes com 0,6 e $1,2 \mathrm{dm}^{3}$ e 7 $\mathrm{h}$ de luminosidade, enquanto que para o recipiente com $3,4 \mathrm{dm}^{3}$, as plantas expostas a luminosidade de $4 \mathrm{~h}$ apresentaram maior largura da folha.

Para a largura da folha (Tabela 4), ocorreu interação significativa entre os fatores, para os dois ciclos estudados, com a análise de regressão sendo mostrada na Figura 3B. Nota-se, que as plantas com $4 \mathrm{e} 7 \mathrm{~h}$ de sol no primeiro ciclo e as plantas do segundo ciclo com $4 \mathrm{~h}$ de luminosidade, apresentaram modelo de regressão linear, com o aumento do volume proporcionando maior largura da folha. Enquanto que na largura da folha das plantas do segundo ciclo, com $7 \mathrm{~h}$ de luminosidade, o modelo de regressão foi o polinomial de segunda ordem, com maior largura $(9,8 \mathrm{~cm})$ para o recipiente com $1,6 \mathrm{dm}^{3}$. 

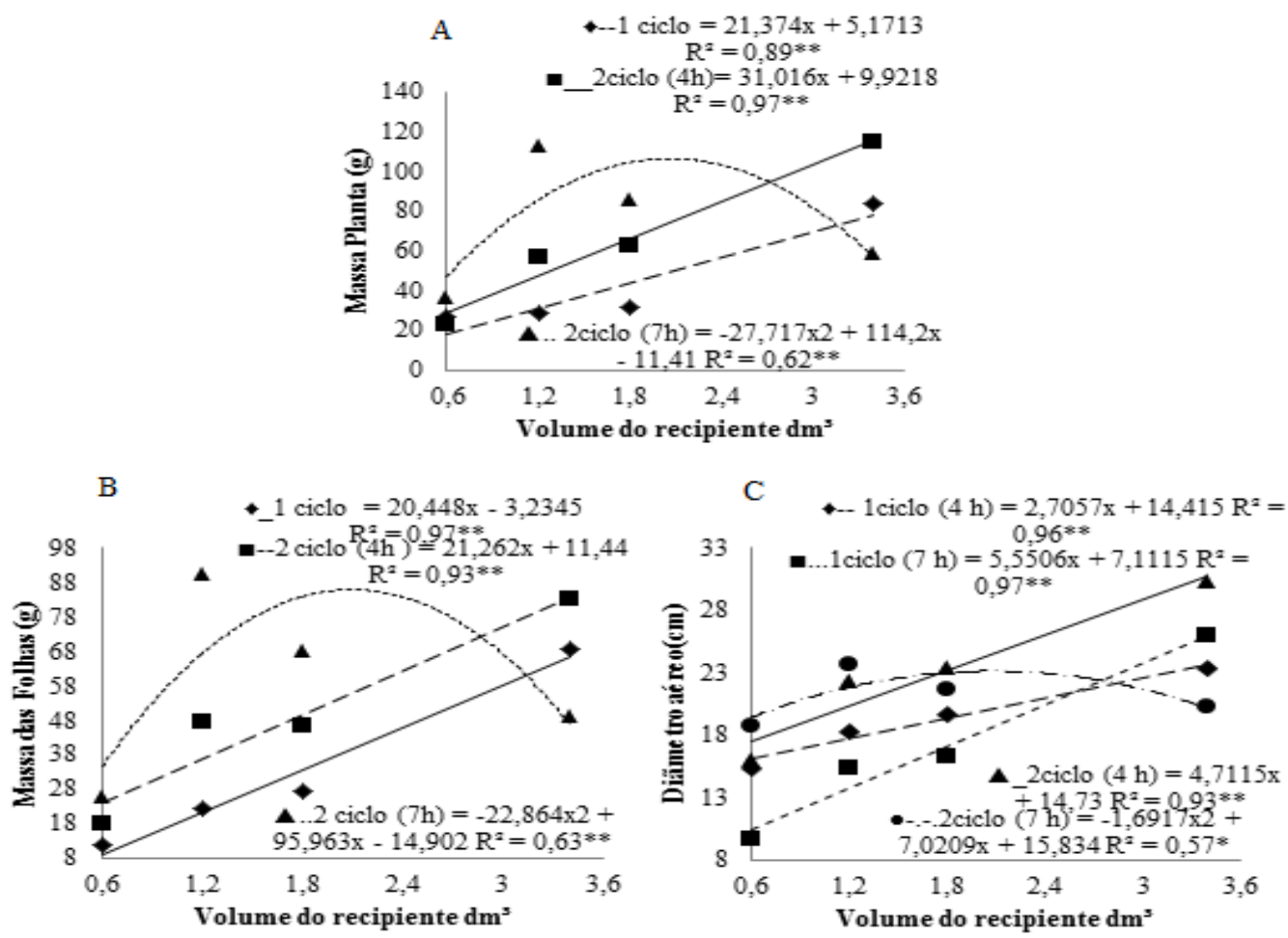

Figura 2. Massa das plantas (A), massa das folhas (B) e diâmetro aéreo (C) de plantas de alface em função do volume do recipiente.

Tabela 4. Resultado da análise de variância e apresentação dos dados médios do fator luminosidade para o comprimento da maior folha $(\mathrm{CF})$, largura da maior folha $(\mathrm{LF})$ e o número de folhas $\left(\mathrm{N}^{\circ} \mathrm{F}\right)$, para o primeiro e segundo ciclo do alface

\begin{tabular}{|c|c|c|c|c|c|c|}
\hline \multirow{3}{*}{ Fontes de Variação } & \multicolumn{6}{|c|}{ Valores de F } \\
\hline & \multicolumn{2}{|c|}{$\mathrm{CF}$} & \multicolumn{2}{|c|}{ LF } & \multicolumn{2}{|c|}{$\mathrm{N}^{\circ} \mathrm{F}$} \\
\hline & $1^{\circ}$ Ciclo & $2^{\circ}$ Ciclo & $1^{\circ}$ Ciclo & $2^{\circ}$ Ciclo & $1^{\circ}$ Ciclo & $2^{\circ}$ Ciclo \\
\hline Luminosidade (L) & $2,91^{\mathrm{NS}}$ & $5,37 *$ & $0,03^{\mathrm{NS}}$ & $0,30^{\mathrm{NS}}$ & $0,50^{\mathrm{NS}}$ & $1,02^{\mathrm{NS}}$ \\
\hline Recipiente (R) & $3,44^{\mathrm{NS}}$ & $7,36 * *$ & $21,43 * *$ & $7,89 * *$ & $2,19^{\mathrm{NS}}$ & $8,77 * *$ \\
\hline $\mathrm{L} * \mathrm{R}$ & $1,48^{\mathrm{NS}}$ & $6,35 * *$ & $3,92 *$ & $17,33 * *$ & $0,57^{\mathrm{NS}}$ & $7,64 * *$ \\
\hline $\mathrm{CV} \%$ & 37,55 & 12,30 & 13,48 & 8,10 & 28,16 & 14,99 \\
\hline \multirow{4}{*}{ Luminosidade (h) } & \multicolumn{6}{|c|}{ Dados Médios } \\
\hline & \multicolumn{2}{|c|}{$\mathrm{CF}$} & \multicolumn{2}{|c|}{$\mathrm{LF}$} & \multicolumn{2}{|c|}{$\mathrm{N}^{\circ} \mathrm{F}$} \\
\hline & $1^{\circ}$ Ciclo & $2^{\circ}$ Ciclo & $1^{\circ}$ Ciclo & $2^{\circ}$ Ciclo & $1^{\circ}$ Ciclo & $2^{\circ}$ Ciclo \\
\hline & \multicolumn{2}{|c|}{-------- cm --------- } & \multicolumn{2}{|c|}{-------- cm --------- } & \multicolumn{2}{|c|}{ Unidade } \\
\hline 4 & 15,1 & - & - & - & 10,7 & - \\
\hline 7 & 11,6 & - & - & - & 9,8 & - \\
\hline
\end{tabular}

**: significativo $(\mathrm{P}<0,01)$; * significativo $(\mathrm{P}<0,05)$; NS: não significativo; CV\%: coeficiente de variação. Células sem valores indicam que ocorreu interação significativa, com os valores sendo apresentados nas tabelas de desdobramento da interação.

Na Tabela 4, observa-se que o comprimento da folha, no $1^{\circ}$ ciclo, não apresentou diferença significativa para os recipientes avaliados, não sendo gerado nenhuma curva de regressão. Entretanto, para o $2^{\circ}$ ciclo (Figura 3A), observa-se que o modelo de regressão foi linear para os tratamentos submetidos à quatro horas de luminosidade, significativo $(\mathrm{p}<0,01)$, ocorrendo comprimento maior da folha com o aumento do volume do recipiente, enquanto que para os tratamentos com sete horas de luminosidade o modelo de regressão foi o polinomial de segunda ordem, apresentando diferença significativa $(p<0,05)$, com o recipiente de $2,0 \mathrm{dm}^{3}$ produzindo folhas maiores $(16,5 \mathrm{~cm})$.

Ao avaliar o número de folhas em função do volume do recipiente, nota-se que o primeiro ciclo não apresentou interação significativa (Tabela 4); apresentando comportamento linear significativo a $5 \%$ de probabilidade (Figura 3C), com aumento no número 
de folhas para os maiores recipientes. Enquanto o segundo ciclo apresentou interação significativa, e com $4 \mathrm{~h}$ de luminosidade, o modelo de regressão foi linear significativo $(p<0,01)$, com o aumento do volume do recipiente promovendo maior número de folhas. Já para $7 \mathrm{~h}$ de luminosidade, o modelo de regressão foi o polinomial de segunda ordem, apresentando diferença significativa a $1 \%$, com 17,8 folhas por planta sendo o valor máximo obtido para o volume de recipiente estimado em $2,1 \mathrm{dm}^{3}$.

Tabela 5. Interação entre os fatores volume do recipiente e luminosidade para a variável comprimento da folha $(\mathrm{cm}) \mathrm{e}$ número de folhas, no segundo ciclo da alface

\begin{tabular}{cccc}
\hline Variável & $\begin{array}{c}\text { Recipiente } \\
\left(\mathrm{dm}^{3}\right)\end{array}$ & \multicolumn{2}{c}{ Luminosidade } \\
\cline { 3 - 4 } & 0,6 & $12,0 \mathrm{a}$ & $13,3 \mathrm{a}$ \\
Comprimento da & 1,2 & $17,0 \mathrm{a}$ & $16,7 \mathrm{a}$ \\
Folha & 1,8 & $16,3 \mathrm{a}$ & $15,7 \mathrm{a}$ \\
& 3,4 & $21,3 \mathrm{a}$ & $13,7 \mathrm{~b}$ \\
& Média & $16,7 \mathrm{~A}$ & $14,8 \mathrm{~B}$ \\
$\mathrm{~N}^{\text {o de Folhas }}$ & 0,6 & $11 \mathrm{a}$ & $10,7 \mathrm{a}$ \\
& 1,2 & $14,3 \mathrm{a}$ & $18,0 \mathrm{a}$ \\
& 1,8 & $15,0 \mathrm{a}$ & $16,0 \mathrm{a}$ \\
& 3,4 & $20,7 \mathrm{a}$ & $12,7 \mathrm{~b}$ \\
\cline { 3 - 4 } & Média & $15,2 \mathrm{~A}$ & $14,3 \mathrm{~A}$ \\
\hline
\end{tabular}

Médias seguidas pela mesma letra nas linhas, não diferem estatisticamente entre si, segundo o teste de TuKey a $5 \%$ de probabilidade.

Tabela 6. Interação entre os fatores volume do recipiente e luminosidade para a variável largura da folha $(\mathrm{cm})$ no primeiro e segundo ciclo da alface.

\begin{tabular}{|c|c|c|c|c|c|}
\hline \multirow{3}{*}{ Variável } & \multirow{3}{*}{$\begin{array}{l}\text { Recipiente } \\
\qquad\left(\mathrm{dm}^{3}\right)\end{array}$} & \multicolumn{4}{|c|}{ Luminosidade } \\
\hline & & \multicolumn{2}{|c|}{$1^{\circ}$ Ciclo } & \multicolumn{2}{|c|}{$2^{\circ}$ Ciclo } \\
\hline & & $4 \mathrm{~h}$ & $7 \mathrm{~h}$ & $4 \mathrm{~h}$ & $7 \mathrm{~h}$ \\
\hline \multirow{5}{*}{$\begin{array}{c}\text { Largura da } \\
\text { Folha }\end{array}$} & 0,6 & $7,3 \mathrm{a}$ & $6,0 \mathrm{a}$ & $6,7 \mathrm{~b}$ & $9,0 \mathrm{a}$ \\
\hline & 1,2 & $10,0 \mathrm{a}$ & $8,3 \mathrm{a}$ & $8,3 \mathrm{~b}$ & $10,3 \mathrm{a}$ \\
\hline & 1,8 & $8,3 \mathrm{a}$ & $9,0 \mathrm{a}$ & $10,0 \mathrm{a}$ & $9,3 \mathrm{a}$ \\
\hline & 3,4 & $11,0 \mathrm{~b}$ & $13,7 \mathrm{a}$ & $11,0 \mathrm{a}$ & $8,0 \mathrm{~b}$ \\
\hline & Média & $9,2 \mathrm{~A}$ & $9,3 \mathrm{~A}$ & $9,0 \mathrm{~A}$ & $9,2 \mathrm{~A}$ \\
\hline
\end{tabular}

Médias seguidas pela mesma letra nas linhas não diferem estatisticamente entre si segundo o teste de TuKey a 5\% de probabilidade.

Resultados semelhantes foram obtidos por Franco et al. (2016), que, avaliando o uso de diferentes recipientes plásticos para o cultivo de alface em espaços urbanos, observaram aumento significativo no comprimento, largura e número de folhas com aumento do volume dos recipientes de 0,6 para $2 \mathrm{dm}^{3}$, com média de 15,$45 ; 8,43$ e 8,75 cm para CF, LF e NF, respectivamente, no menor recipiente e de 18,$30 ; 11,65$ e $15 \mathrm{~cm}$ para $C F, L F N^{\circ} F$ no maior recipiente.

$\mathrm{Na}$ Tabela 7, o coeficiente de variação para a variável diâmetro do caule foi alto e médio no $1^{\circ}$ e $2^{\circ}$ ciclo respectivamente, enquanto que para o comprimento do caule ambos os ciclos obtiveram cv muito alto segundo Pimentel Gomes (2009). Observa-se que para a luminosidade não ocorreram resultados significativos para o diâmetro e comprimento do caule. Resultados semelhantes foram obtidos por Brzezinski et al. (2017), entretanto, Diamante et al. (2013), avaliando o ambiente no cultivo de alface, verificaram diferença estatística para os ambientes, com a menor média de comprimento de caule em ambiente sem sombreamento $(14,7 \mathrm{~cm})$ e de $21,2 \mathrm{~cm}$, com sombreamento.

Luz et al. (2009) obtiveram os menores comprimentos de caule no campo aberto e maiores nos ambientes com tela de sombreamento, ambos trabalhos com valores superiores aos deste. Tais resultados foram provavelmente, em função da cultivar utilizada pelos autores, aliado ao período de colheita após a semeadura, 15 dias após que o do presente estudo, com estes altos valores podendo ser aferidos ao início do pendoamento da alface, em função do atraso na colheita. Maiores valores de comprimento e diâmetro do caule são indesejados pois depreciam a qualidade do produto, perdendo valor de mercado.

Foi observada interação significativa para o diâmetro no caule, no segundo ciclo, entre as horas de luminosidade e o volume do recipiente, com essa interação sendo desdobrada na Tabela 8 .

Na Tabela 8, o recipiente com 1,2 $\mathrm{dm}^{3}$ de volume, proporcionou maior diâmetro do caule para as plantas submetidas a 7 horas de luminosidade, enquanto que para os recipientes com volume de $3,4 \mathrm{dm}^{3}$, as plantas obtiveram maior diâmetro do caule quando expostas a 4 horas de luminosidade. Nota-se, que o maior recipiente proporcionou maior comprimento de caule quando submetido à menor luminosidade; resultados semelhantes foram obtidos por Neves et al. (2016), avaliando quatro variedades de alface em canteiros (grande volume de solo), com maior comprimento do caule para o cultivo em ambiente aberto (máxima luminosidade).

Em relação ao volume do recipiente, para o diâmetro do caule (Figura $4 \mathrm{~A}$ ), verifica-se que no primeiro ciclo não ocorreu interação significativa entre os fatores, e para o segundo ciclo, com $4 \mathrm{~h}$ de luminosidade, com interação significativa (Tabela 7), com o aumento do volume do recipiente proporcionando maior diâmetro do caule. Já no diâmetro do caule do segundo ciclo, mas com 7 horas de luminosidade, o modelo de regressão foi o polinomial de segunda ordem, com maior diâmetro $(1,4 \mathrm{~cm})$ para o recipiente com $2,1 \mathrm{dm}^{3}$. 
A

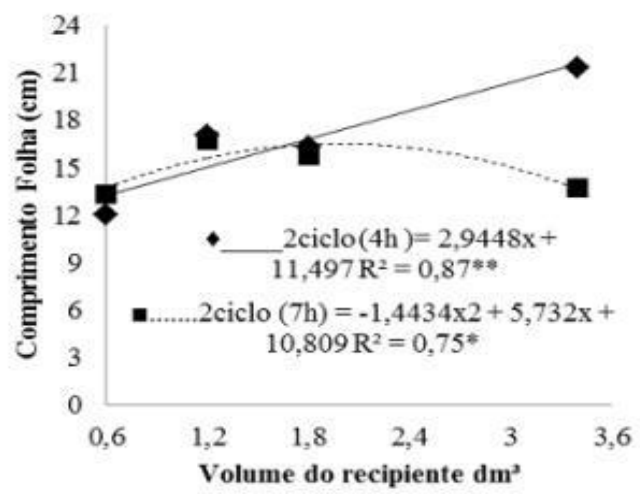

B

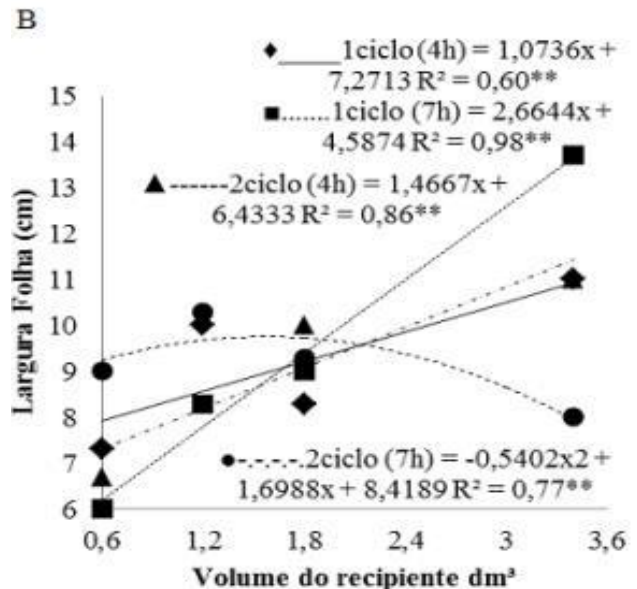

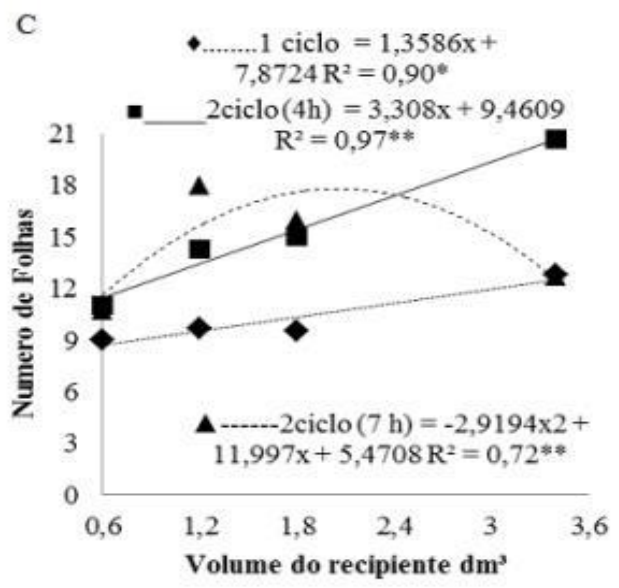

Figura 3. Comprimento da folha (A), largura da folha (B) e número de folhas (C) de alface em função do volume do recipiente.

Tabela 7. Resultado da análise de variância e apresentação dos dados médios do fator luminosidade para o diâmetro do caule (DC), e comprimento do caule (CC), para o primeiro e segundo ciclo da alface.

\begin{tabular}{|c|c|c|c|c|}
\hline \multirow{3}{*}{ Fontes de Variação } & \multicolumn{4}{|c|}{ Valores de F } \\
\hline & \multicolumn{2}{|c|}{ DC } & \multicolumn{2}{|c|}{$\mathrm{CC}$} \\
\hline & $1^{\circ}$ Ciclo & $2^{\circ}$ Ciclo & $1^{\circ}$ Ciclo & $2^{\circ}$ Ciclo \\
\hline $\begin{array}{c}\text { Luminosidade (I) } \\
\text { Recipiente (R) } \\
\text { I*R }\end{array}$ & $\begin{array}{c}0,41^{\mathrm{NS}} \\
17,07^{* *} \\
1,29^{\mathrm{NS}} \\
\end{array}$ & $\begin{array}{c}2,74^{\mathrm{NS}} \\
14,28 * * \\
10,58 * *\end{array}$ & $\begin{array}{c}2,59^{\mathrm{NS}} \\
12,38^{* *} \\
0,65^{\mathrm{NS}} \\
\end{array}$ & $\begin{array}{l}0,011^{\mathrm{NS}} \\
9,33 * * \\
14,10^{\mathrm{NS}}\end{array}$ \\
\hline $\mathrm{CV} \%$ & 25,63 & 17,96 & 41,97 & 41,17 \\
\hline \multirow{4}{*}{ Luminosidade (h) } & \multicolumn{4}{|c|}{ Dados Médios } \\
\hline & \multicolumn{2}{|c|}{ DC } & \multicolumn{2}{|c|}{$\mathrm{CC}$} \\
\hline & $1^{\circ}$ Ciclo & $2^{\circ}$ Ciclo & $1^{\circ}$ Ciclo & $2^{\circ}$ Ciclo \\
\hline & \multicolumn{2}{|c|}{----------------- cm ------------------ } & \multicolumn{2}{|c|}{ 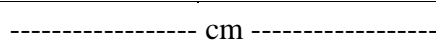 } \\
\hline 4 & 0,96 & - & 2,6 & 5,3 \\
\hline 7 & 1,03 & - & 2,0 & 5,2 \\
\hline
\end{tabular}

**: significativo $(\mathrm{P}<0,01)$; *: significativo $(\mathrm{P}<0,05)$; NS: não significativo; $\mathrm{CV} \%$ : coeficiente de variação. Células sem valores indicam que ocorreu interação significativa, com os valores sendo apresentados nas tabelas de desdobramento da interação.

Para o comprimento do caule, não ocorreram interações significativas nos ciclos avaliados (Tabela 7). Verifica-se na Figura 4 B, que o modelo de regressão foi linear para os dois ciclos, com maior comprimento de caule com o aumento do volume do recipiente. Para a massa (Tabela 9), não ocorreu diferença estatística em ambos os ciclos estudados; tais valores foram inferiores aos obtidos por Baumgartner et al. (2007) 
ao verificarem o desenvolvimento de alface no estado do Paraná, para o comprimento da raiz, com média de $12,8 \mathrm{~cm}$ e médias da massa de raiz $(11,8 \mathrm{~g})$.

Tabela 8. Interação entre os fatores volume do recipiente e luminosidade para a variável diâmetro do caule $(\mathrm{cm})$ no segundo ciclo da alface.

\begin{tabular}{cccc}
\hline \multirow{2}{*}{ Variável } & \multirow{2}{*}{ Recipiente $\left(\mathrm{dm}^{3}\right)$} & \multicolumn{2}{c}{ Luminosidade } \\
\cline { 2 - 4 } & & $4 \mathrm{~h}$ & $7 \mathrm{~h}$ \\
\hline \multirow{2}{*}{ Diâmetro do } & 0,6 & $0,47 \mathrm{a}$ & $0,77 \mathrm{a}$ \\
caule & 1,2 & $0,73 \mathrm{~b}$ & $1,40 \mathrm{a}$ \\
& 1,8 & $1,17 \mathrm{a}$ & $1,20 \mathrm{a}$ \\
& 3,4 & $1,50 \mathrm{a}$ & $1,00 \mathrm{~b}$ \\
\cline { 2 - 4 } & Média & $0,97 \mathrm{~A}$ & $1,09 \mathrm{~A}$ \\
\hline
\end{tabular}

Médias seguidas pela mesma letra nas linhas não diferem estatisticamente entre si, segundo o teste de Tukey a $5 \%$ de probabilidade.

Na Tabela 9, o comprimento da raiz obteve interação significativa entre os fatores estudados, nos dois ciclos, com tal interação sendo desdobrada na Tabela 10 e Figura 5. Os tratamentos com $1,2 \mathrm{dm}^{3}$ de substrato promoveram aumento no comprimento da raiz, no $1^{\circ} \mathrm{e}$ $2^{\circ}$ ciclo, quando as plantas obtiveram luminosidade diária de quatro horas, bem como os tratamentos com $0,6 \mathrm{dm}^{3}$ de solo, no segundo ciclo, que obtiveram maior comprimento da raiz quando a luminosidade diária foi de 4 h. Em relação ao volume do recipiente (Figura 5 A), o comprimento da raiz obteve modelo de regressão polinomial de segunda ordem significativo, para os dois ciclos, em ambas as insolações estudadas.

$\mathrm{Na}$ Figura $5 \mathrm{~A}$, nota-se que a massa da raiz em função do volume do recipiente, apresentou modelo de regressão linear para o primeiro ciclo, e polinomial de segunda ordem para o segundo ciclo, ambos significativos $(\mathrm{p}<0,01)$, com maior massa de raízes $(8,7$ $\mathrm{cm}$ ) para o recipiente com $2,4 \mathrm{dm}^{3}$ de volume
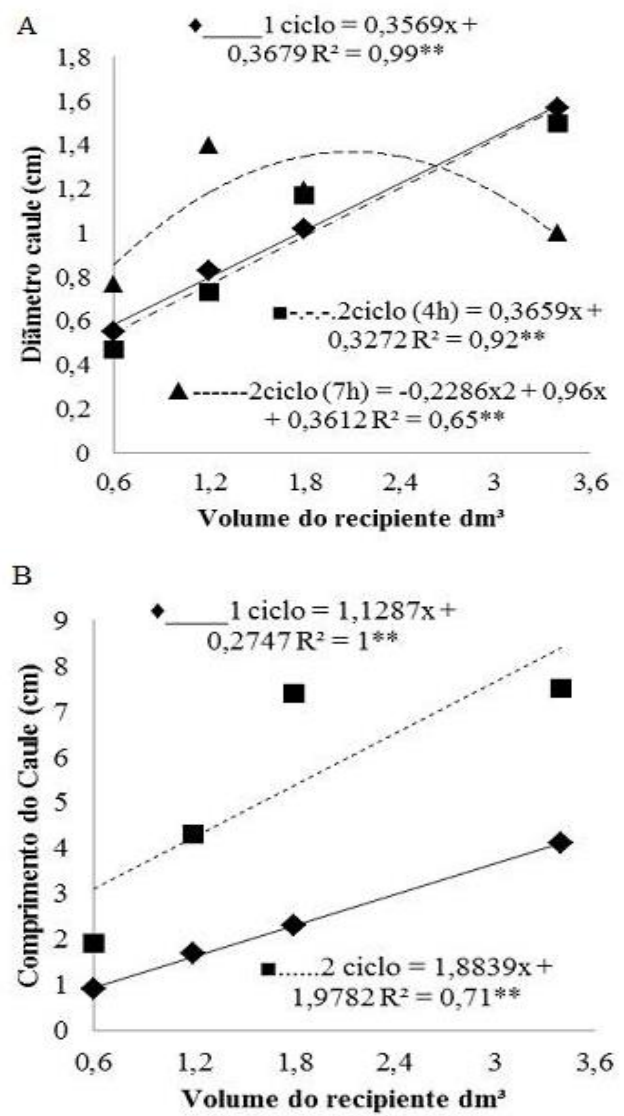

Figura 4. Diâmetro do caule (A) e comprimento do caule (B) das plantas de alface em função do volume do recipiente.

Tabela 9. Resultado da análise de variância e apresentação dos dados médios do fator luminosidade para o comprimento da raiz (CR), e massa da raiz (MR) para o primeiro e segundo ciclo da alface

\begin{tabular}{|c|c|c|c|c|}
\hline \multirow{3}{*}{ Fontes de Variação } & \multicolumn{4}{|c|}{ Valores de F } \\
\hline & \multicolumn{2}{|c|}{$\mathrm{CR}$} & \multicolumn{2}{|c|}{ MR } \\
\hline & $1^{\circ}$ Ciclo & $2^{\circ}$ Ciclo & $1^{\circ}$ Ciclo & $2^{\circ}$ Ciclo \\
\hline I & $0,09^{\mathrm{NS}}$ & $17,19 * *$ & $0,33^{\mathrm{NS}}$ & $0,18^{\mathrm{NS}}$ \\
\hline $\mathrm{R}$ & $7,83 * *$ & $11,82 * *$ & $7,19 * *$ & $17,09 * *$ \\
\hline $\mathrm{I} * \mathrm{R}$ & $6,70 *$ & $4,17 *$ & $1,69^{\mathrm{NS}}$ & $0,41^{\mathrm{NS}}$ \\
\hline $\mathrm{CV} \%$ & 20,01 & 16,92 & 44,45 & 21,70 \\
\hline \multirow{4}{*}{ Luminosidade (h) } & \multicolumn{4}{|c|}{ Dados Médios } \\
\hline & \multicolumn{2}{|c|}{$\mathrm{CR}$} & \multicolumn{2}{|c|}{ MR } \\
\hline & $1^{\circ}$ Ciclo & $2^{\circ}$ Ciclo & $1^{\circ}$ Ciclo & $2^{\circ}$ Ciclo \\
\hline & \multicolumn{2}{|c|}{---------- cm ---------- } & \multicolumn{2}{|c|}{ 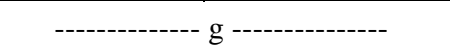 } \\
\hline 4 & - & - & 6,2 & 6,1 \\
\hline 7 & - & - & 5,6 & 6,3 \\
\hline
\end{tabular}


Tabela 10. Interação entre os fatores volume do recipiente e luminosidade para a variável comprimento da raiz $(\mathrm{cm})$, no primeiro e segundo ciclo da alface.

\begin{tabular}{|c|c|c|c|c|}
\hline \multirow{3}{*}{$\begin{array}{l}\text { Recipiente } \\
\qquad\left(\mathrm{dm}^{3}\right)\end{array}$} & \multicolumn{4}{|c|}{ Luminosidade } \\
\hline & \multicolumn{2}{|c|}{$1^{\circ}$ Ciclo } & \multicolumn{2}{|c|}{$2^{\circ}$ Ciclo } \\
\hline & $4 \mathrm{~h}$ & $7 \mathrm{~h}$ & $4 \mathrm{~h}$ & $7 \mathrm{~h}$ \\
\hline 0,6 & $4,0 \mathrm{a}$ & $5,3 \mathrm{a}$ & $8,0 \mathrm{a}$ & $5,0 \mathrm{~b}$ \\
\hline 1,2 & $9,0 \mathrm{a}$ & $4,7 \mathrm{~b}$ & $10,3 \mathrm{a}$ & $6,3 \mathrm{~b}$ \\
\hline 1,8 & $7,7 \mathrm{a}$ & $9,0 \mathrm{a}$ & $8,7 \mathrm{a}$ & $7,3 \mathrm{a}$ \\
\hline 3,4 & $6,0 \mathrm{a}$ & $7,0 \mathrm{a}$ & $4,5 \mathrm{a}$ & $5,0 \mathrm{a}$ \\
\hline Média & $6,7 \mathrm{~A}$ & $6,5 \mathrm{~A}$ & $7,9 \mathrm{~A}$ & $5,9 \mathrm{~B}$ \\
\hline
\end{tabular}

Médias seguidas pela mesma letra minúscula nas fileiras não diferem estatisticamente entre si segundo o teste de Tukey a $5 \%$ de probabilidade.

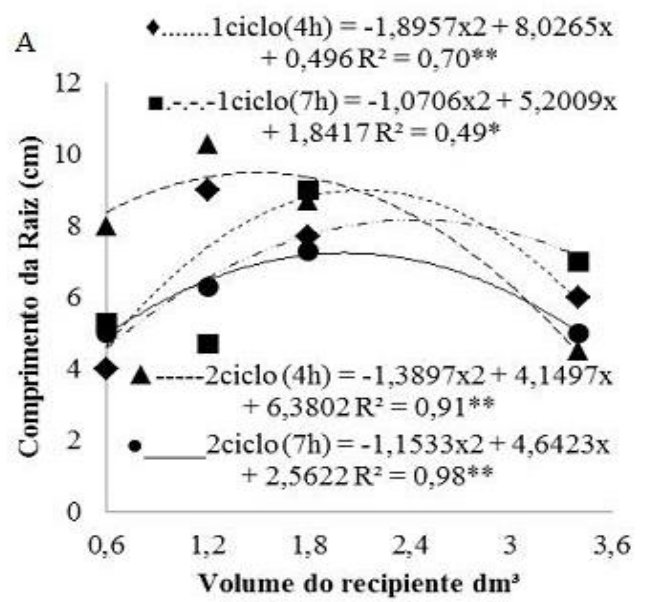

B

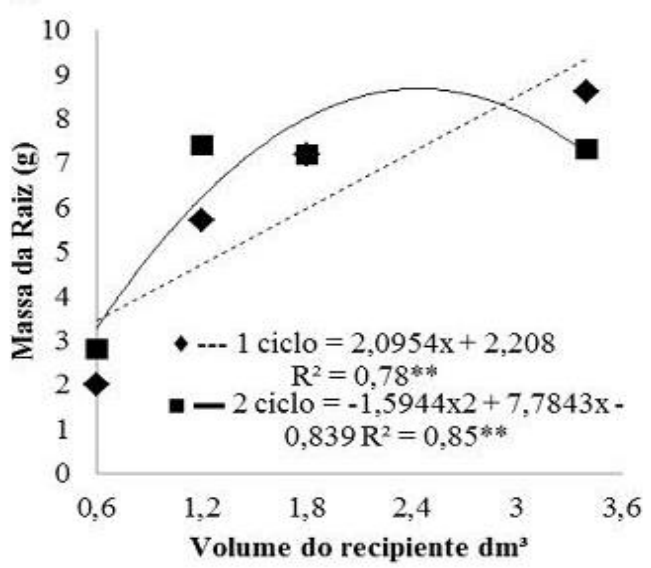

Figura 5. Comprimento da raiz (A) e massa da raiz (B) das plantas de alface em função do volume dos recipientes.

\section{Conclusões}

Para o primeiro ciclo da cultura, a alface apresentou melhor desenvolvimento para os recipientes com maior volume $\left(3,4 \mathrm{dm}^{3}\right)$, sem interferência da luminosidade diária.

No segundo ciclo, com incidência solar diária de $4 \mathrm{~h}$, obteve o melhor resultado para o maior recipiente estudado. Entretanto, para $7 \mathrm{~h}$ de luminosidade, os melhores resultados foram obtidos para o recipiente com volume de $2,1 \mathrm{dm}^{3}$.
A luminosidade diária teve efeito no segundo ciclo da cultura, com melhores resultados para a menor luminosidade (4 h).

$\mathrm{O}$ cultivo de alface, em recipientes maiores $(>2$ $\mathrm{dm}^{3}$ ) obteve bons resultados, sendo viável a produção de alface com luminosidade de 4 h diárias.

\section{Agradecimentos}

Ao Programa de Educação Tutorial do curso de Agronomia (PET Agro) da Universidade Federal do Cariri (UFCA) pela concessão de bolsas de estudo, que tanto contribuíram para a execução desta pesquisa.

\section{Referências bibliográficas}

Baumgartner, D.; Sampaio, S.C.; Silva, T.; Teo, C.R.P.A.; Vilas Boas, M.A., 2007. Reuso de águas residuárias da piscicultura e da suinocultura na irrigação da cultura da alface. Engenharia Agrícola, 27, 152-163.

Blind, D.A.; Silva Filho, D.F., 2015. Desempenho produtivo de cultivares de alface americana na estação seca da amazônia central. Bioscience Journal, 31, 404-414.

Brzezinski, C.R.; Abati, J.; Geller, A.; Werner, F.; Zucareli, C., 2017. Produção de cultivares de alface americana sob dois sistemas de cultivo. Revista Ceres, 64, 83-89.

Dalastra, M.G.; Hachmann, T.L.; Echer, M.M.; Guimarães, F.V.; Fiametti, M.S., 2016. Características produtivas de cultivares de alface mimosa, conduzida sob diferentes níveis de sombreamento, no inverno. Scientia Agraria Paranaensis, $15,15-19$.

Diamante, M.S.; Seabra Júnior, S.; Inagaki, A.M.; Silva, M.B.; Dallacort, R., 2013. Produção e resistência ao pendoamento de alfaces tipo lisa cultivadas sob diferentes ambientes. Revista Ciência Agronômica, 44, 133-140.

Dias-Filho, M.B., 1997. Physiological response of Solanum crinitum Lam. To contrasting light environments. Pesquisa Agropecuária Brasileira, 32, 789-796.

Flôres, J.A.; Santos, L.A.C.; Silva, D.M.P.; Oliveira, I.A.; Pereira, C.E., 2016. Desempenho agronômico de cultivares de alface em casa de vegetação no município de Humaitá, AM. Revista Ciência Agroambientais, 14, 113-116.

Franco, H.A.; Jorge, E.N.L.F.; Pereira, W.M.; Sampaio, F.P.; Thode Filho, S.; Carvalho, M.D., 2016. Gestão de resíduos pós-consumo: avaliação de recipientes para produção de alface (Lactuca sativa L.) em horta urbana. Educação, Gestão e Sociedade, 7, 1-8.

Ferreira, D.F. SISVAR: um computador sistema de análise estatística., 2011. Ciência e Agrotecnologia, 35, 1039-1042.

Filgueira, F.A.R., 2013. Novo manual de olericultura: agrotecnologia moderna na produção e comercialização de hortaliças, UFV, Viçosa. 
Funceme. Fundação Cearense de Meteorologia e Recursos Hídricos. 20012 Levantamento de reconhecimento de média intensidade dos solos da Mesoregião do Sul Cearense, FUNCEME, Fortaleza.

Holt, J.S., 1995. Plant response to light: a potencial tool for weed management. Weed Science, 43, 474-482.

Lima G.G.; Ribeiro S.C., 2012. Geomorfologia e paisagem do município de Juazeiro do Norte/CE: relações entre a natureza semiárida e os impactos antrópicos. Revista Geonorte, 2, 520530.

Luz, A.O.; Seabra Júnior, S.; Souza, S.B.S.; Nascimento, A.S., 2009. Resistência ao pendoamento de genótipos de alface em ambientes de cultivo. Agrarian, 2, 71-82.

Monteiro, D.; Mendonça, M.M., 2004. Quintais na cidade: a experiência de 1011 moradores da periferia do Rio de Janeiro. Revista Agriculturas: experiências em agroecologia, 1, 29-31.

Moreira M.A.; Santos C.A. P; Lucas A.A.T; Bianchini F.G.; Souza I.M.; Viégas P.R.A. 2014. Lettuce production according to different sources of organic matter and soil cover. Agricultural Sciences, 5, 99105.
Neves, J.F.; Nodari, I.D.E.; Júnior, S.S.; Dias, L.D.E.; Silva, L.B.; Dallacort, R. 2014. Produção de cultivares de alface americana sob diferentes ambientes em condições tropicais. Revista Agro@mbiente, 10, 130-136.

Pimentel Gomes, F., 2009. Curso de estatística experimental. 15. ed. FEALQ, Piracicaba.

Santana, C.V.S.; Almeida, A.C.; Turco, S.H.N., 2009. Produção de alface roxa em ambientes sombreados na região do submédio São Francisco - BA. Revista Verde, 4, 1-6.

Silva, E.M.C.P.; Ferreira, R.L.F.; Ribeiro, A.M.A.S.; Neto, S.E.A.N.; Kusdra, J.F., 2015. Desempenho agronômico de alface orgânica influenciado pelo sombreamento, época de plantio e preparo do solo no Acre. Pesquisa Agropecuária Brasileira, Brasília, 50, 468-474.

Silva, A.R.; Forlan, P.V.; Ferrari, Samuel, F.; Pereira, M.G.P. 2014. Telas de sombreamento no desempenho de cultivares de alface. Núcleos, 11, 433-442. 